\title{
Gribov horizon and BRST symmetry: a few remarks
}

\author{
S.P. Sorella ${ }^{* \dagger}$ \\ UERJ, Universidade do Estado do Rio de Janeiro, \\ Instituto de Física, \\ Departamento de Física Teórica, \\ Rua São Francisco Xavier 524, \\ 20550-013 Maracanã, Rio de Janeiro, Brasil
}

October 31, 2018

\begin{abstract}
The issue of the BRST symmetry in presence of the Gribov horizon is addressed in Euclidean Yang-Mills theories in the Landau gauge. The positivity of the Faddeev-Popov operator within the Gribov region enables us to convert the soft breaking of the BRST invariance exhibited by the Gribov-Zwanziger action into a non-local exact symmetry, displaying explicit dependence from the non-perturbative Gribov parameter. Despite its non-locality, this symmetry turns out to be useful in order to establish non-perturbative Ward identities, allowing us to evaluate the vacuum expectation value of quantities which are BRST exact. These results are generalized to the refined Gribov-Zwanziger action introduced in [1, which yields a gluon propagator which is non-vanishing at the origin in momentum space, and a ghost propagator which is not enhanced in the infrared.
\end{abstract}

*sorella@uerj.br

†Work supported by FAPERJ, Fundação de Amparo à Pesquisa do Estado do Rio de Janeiro, under the program Cientista do Nosso Estado, E-26/100.615/2007. 


\section{Introduction}

The BRST symmetry is a fundamental tool for the study of gauge theories. This symmetry is at the origin of the Slavnov-Taylor identities which guarantee the renormalizability of Yang-Mills theories [2]. Furthermore, the use of the BRST charge enables us to identify the physical subspace of the theory, allowing to prove the unitarity of the $S$-matrix 1 . Though, reconciling this symmetry with the appearance of non-perturbative effects represents a non-trivial challenge in quantum field theory.

In particular, the study of the BRST symmetry when the Gribov copies [3] are taken into account is of great relevance in order to unravel the non-perturbative features of confining Yang-Mills theories [4, 5. While the Gribov copies can be neglected in the perturbative ultraviolet regime, they play a relevant role in the infrared, being closely related to gluon confinement. A partial resolution of the Gribov issue is provided by the Gribov-Zwanziger framework [3, 6, 7], amounting to restrict the domain of integration in the functional integral to the Gribov region, whose boundary is the first Gribov horizon. Although the Gribov region is not completely free from Gribov copies [8], the restriction to this region can be achieved by adding to the Yang-Mills action a non-local term $S_{h}$, known as the horizon function [6, 7]

$$
\begin{aligned}
S_{h} & =\gamma^{4} \int d^{4} x h(x), \\
h(x) & =g^{2} f^{a b c} A_{\mu}^{b}\left(\mathcal{M}^{-1}\right)^{a d} f^{d e c} A_{\mu}^{e},
\end{aligned}
$$

where $\left(\mathcal{M}^{-1}\right)^{a d}$ is the inverse of the Faddeev-Popov operator $\mathcal{M}^{a b}=-\partial_{\mu}\left(\partial_{\mu} \delta^{a b}+g f^{a c b} A_{\mu}^{c}\right)$. The massive parameter $\gamma$ appearing in eq.(11) is the Gribov parameter [3]. It is not a free parameter, being determined in a self-consistent way as a function of the gauge coupling constant $g$ and of the invariant scale $\Lambda_{Q C D}$ through the so called horizon condition [6, 7]

$$
\langle h(x)\rangle=4\left(N^{2}-1\right) .
$$

Despite its non-locality, the horizon function can be cast in local form by means of a set of auxiliary fields, $\left(\bar{\varphi}_{\mu}^{a b}, \varphi_{\mu}^{a b}, \bar{\omega}_{\mu}^{a b}, \omega_{\mu}^{a b}\right)$, see Sect.3. A local action is thus obtained from the Gribov horizon. Remarkably, it enjoys the important property of being renormalizable [6, 17, 19, 10, 11, 12].

Having at our disposal a local renormalizable action encoding the restriction to the Gribov region, we can ask ourselves whether it possesses BRST invariance. Here, we need to be more precise about the question we are addressing. Till now, the BRST transformations we are referring to are those corresponding to the Faddeev-Popov Lagrangian with the inclusion of the auxiliary fields needed to localize the horizon function. As pointed out in [6, 7], these fields give rise to a BRST quartet. Thus, we are considering the following BRST operator $s$ :

$$
\begin{aligned}
s A_{\mu}^{a} & =-D_{\mu}^{a b} c^{b}, & s c^{a} & =\frac{1}{2} g f^{a b c} c^{b} c^{c}, \\
s \bar{c}^{a} & =b^{a}, & s b^{a} & =0, \\
s \varphi_{\mu}^{a b} & =\omega_{\mu}^{a b}, & s \omega_{\mu}^{a b} & =0, \\
s \bar{\omega}_{\mu}^{a b} & =\bar{\varphi}_{\mu}^{a b}, & s \bar{\varphi}_{\mu}^{a b} & =0,
\end{aligned}
$$

\footnotetext{
${ }^{1}$ Here, we are referring to theories for which the asymptotic fields can be consistently introduced.
} 
where $b^{a}$ is the Lagrange multiplier enforcing the gauge condition and $\left(\bar{c}^{a}, c^{a}\right)$ stand for the Faddeev-Popov ghosts. As a first answer to the previous question, one can check if the BRST transformations (3) leave the Gribov-Zwanziger action invariant. This is not the case. It turns out that the Gribov-Zwanziger action does not exhibit invariance under the BRST transformations (3), which are broken by the presence of the horizon 2 . Nevertheless, the resulting breaking term displays peculiar features. It is proportional to the Gribov parameter $\gamma$, being thus a soft breaking. As such, it can be neglected in the deep ultraviolet region, where the notion of exact BRST symmetry is recovered. Moreover, it can be kept under control at the quantum level, due to the possibility of deriving generalized softly broken Slavnov-Taylor identities ensuring the renormalizability of the Gribov-Zwanziger action.

In [11, particular attention was devoted to the auxiliary fields $\left(\bar{\varphi}_{\mu}^{a b}, \varphi_{\mu}^{a b}, \bar{\omega}_{\mu}^{a b}, \omega_{\mu}^{a b}\right)$ which, as much as the Faddeev-Popov ghosts $\left(\bar{c}^{a}, c^{a}\right)$, develop their own dynamics, giving rise to non-perturbative effects. We quote, for example, the dimension two condensate $\left\langle 0\left|\left(\bar{\varphi}_{\mu}^{a b}(x) \varphi_{\mu}^{a b}(x)-\bar{\omega}_{\mu}^{a b}(x) \omega_{\mu}^{a b}(x)\right)\right| 0\right\rangle$, easily written as the vacuum expectation value of a BRST exact quantity, i.e.

$$
\left\langle 0\left|\left(\bar{\varphi}_{\mu}^{a b}(x) \varphi_{\mu}^{a b}(x)-\bar{\omega}_{\mu}^{a b}(x) \omega_{\mu}^{a b}(x)\right)\right| 0\right\rangle=\left\langle 0\left|s\left(\bar{\omega}_{\mu}^{a b}(x) \varphi_{\mu}^{a b}(x)\right)\right| 0\right\rangle .
$$

Without the restriction to the Gribov region, such an expectation value would vanish. However, in the presence of the horizon, and thus for a non-vanishing Gribov parameter $\gamma$, it does not necessarily vanish. The explicit one-loop evaluation of expression (4) shows in fact that it is proportional to the Gribov parameter [11]:

$$
\left\langle 0\left|\left(\bar{\varphi}_{\mu}^{a b}(x) \varphi_{\mu}^{a b}(x)-\bar{\omega}_{\mu}^{a b}(x) \omega_{\mu}^{a b}(x)\right)\right| 0\right\rangle=\frac{3 \sqrt{2}}{64 \pi} g \sqrt{N}\left(N^{2}-1\right) \gamma^{2}
$$

That the vacuum expectation value of quantities which are BRST exact is non-vanishing has a simple understanding, due to the presence of a boundary in field space, namely, the Gribov horizon. We observe that the nilpotent BRST operator (3) can be considered as an exterior derivative along the gauge orbit. However, even if the BRST exact quantity $s\left(\bar{\omega}_{\mu}^{a b} \varphi_{\mu}^{a b}\right)$ can be seen as a total derivative, its integral, i.e. $\left\langle 0\left|s\left(\bar{\omega}_{\mu}^{a b} \varphi_{\mu}^{a b}\right)\right| 0\right\rangle$, picks up a boundary contribution, encoded in the explicit dependence of $\left\langle 0\left|\left(\bar{\varphi}_{\mu}^{a b} \varphi_{\mu}^{a b}-\bar{\omega}_{\mu}^{a b} \omega_{\mu}^{a b}\right)\right| 0\right\rangle$ from the Gribov parameter. The existence of a soft breaking of the BRST symmetry plays an important role here. As noticed in [11], this breaking ensures that the parameter $\gamma$ is a physical parameter of the theory, entering thus the expression of the correlation functions of the theory.

The analysis of the soft BRST breaking done in [11] has also revealed that the possibility of achieving an exact invariance through a suitable modification of the BRST operator by local $\gamma$ dependent terms has to be ruled out, due to the strong constraints imposed by dimensionality of the fields, Lorentz covariance and $S U(N)$ color structure. Nevertheless, we cannot disregard the existence of a non-perturbative non-local symmetry of the Gribov-Zwanziger action, which would be compatible with the presence of the Gribov horizon. This is the issue we aim to face in this work. More specifically, we point out that the soft breaking of the BRST symmetry displayed by the Gribov-Zwanziger action can be converted into a non-local exact invariance. In other words, we shall be able to show that the BRST operator $s$ and its soft breaking can be replaced by an operator $s_{\gamma}$ which corresponds to an exact invariance of the Gribov-Zwanziger action, while

\footnotetext{
${ }^{2}$ See Chapter V of [11] for a detailed discussion on the soft breaking of the BRST symmetry.
} 
exhibiting an explicit dependence from the Gribov parameter $\gamma$. Albeit the operator $s_{\gamma}$ is nonlocal, it can be employed to investigate the dynamics of the auxiliary fields $\left(\bar{\varphi}_{\mu}^{a b}, \varphi_{\mu}^{a b}, \bar{\omega}_{\mu}^{a b}, \omega_{\mu}^{a b}\right)$. We observe in fact that, imposing the condition

$$
\left\langle 0\left|s_{\gamma} \Theta(x)\right| 0\right\rangle=0
$$

where $\Theta(x)$ stands for a local field polynomial depending on the auxiliary fields $\left(\bar{\varphi}_{\mu}^{a b}, \varphi_{\mu}^{a b}, \bar{\omega}_{\mu}^{a b}, \omega_{\mu}^{a b}\right)$, will provide us a way to evaluate the BRST exact quantity $\langle 0|s \Theta(x)| 0\rangle$. For example, from the condition $\left\langle 0\left|s_{\gamma}\left(\bar{\omega}_{\mu}^{a b}(x) \varphi_{\mu}^{a b}(x)\right)\right| 0\right\rangle=0$, we shall be able to obtain the expression of the condensate $\left\langle 0\left|\left(\bar{\varphi}_{\mu}^{a b}(x) \varphi_{\mu}^{a b}(x)-\bar{\omega}_{\mu}^{a b}(x) \omega_{\mu}^{a b}(x)\right)\right| 0\right\rangle$. Of course, the value obtained for $\left\langle 0\left|\left(\bar{\varphi}_{\mu}^{a b} \varphi_{\mu}^{a b}-\bar{\omega}_{\mu}^{a b} \omega_{\mu}^{a b}\right)\right| 0\right\rangle$ from condition (6) coincides with that which has been found by direct calculations [11].

We also underline that condition (6) turns out to be compatible with the gap equation (2) determining the Gribov parameter $\gamma$. As we shall see, from the horizon condition (2) it follows that

$$
\left\langle 0\left|s_{\gamma}\left(g f^{a b c} A_{\mu}^{a}(x) \bar{\omega}_{\mu}^{b c}(x)\right)\right| 0\right\rangle=0,
$$

which is a particular case of condition (6).

The paper is organized as follows. In Sect.2, before facing the more complex case of the GribovZwanziger action, we construct a toy model exhibiting soft breaking of the BRST invariance and reproducing features of the Gribov-Zwanziger action. This model will be employed to show how the operator $s_{\gamma}$ can be introduced and how condition (6) enables us to evaluate the vacuum expectation value of BRST exact quantities. The Noether current corresponding to the exact $s_{\gamma}$-invariance will be derived. Sect.3 is devoted to the Gribov-Zwanziger action. We introduce the operator $s_{\gamma}$ and use it in order to evaluate the condensate $\left\langle 0\left|\left(\bar{\varphi}_{\mu}^{a b} \varphi_{\mu}^{a b}-\bar{\omega}_{\mu}^{a b} \omega_{\mu}^{a b}\right)\right| 0\right\rangle$. The relationship between condition (6) and the gap equation (2) is also discussed. In Sect.4 we generalize the operator $s_{\gamma}$ to the so called refined Gribov-Zwanziger model [1, 11], in which the operator $\left(\bar{\varphi}_{\mu}^{a b} \varphi_{\mu}^{a b}-\bar{\omega}_{\mu}^{a b} \omega_{\mu}^{a b}\right)$ is taken into account from the beginning. In Sect.5 we collect our conclusion.

\section{A toy model and its BRST soft breaking}

Let us start by considering a scalar field $\phi$ whose dynamics is described by the Euclidean action

$$
S_{\phi}=\int d^{4} x\left(\frac{1}{2} \phi\left(-\partial^{2}\right) \phi+\frac{\lambda}{4 !} \phi^{4}\right) .
$$

We look at a mechanism allowing us to modify in a smooth way the behavior of the correlation functions of the theory in the infrared, while leaving the ultraviolet behavior unmodified. As pointed out in [13], this goal can be achieved by the mechanism of the soft breaking of the BRST symmetry. To that purpose, we introduce a $\operatorname{BRST}$ quartet $(\bar{\varphi}, \varphi, \bar{\omega}, \omega)$

$$
\begin{array}{ll}
s \varphi=\omega, & s \omega=0 \\
s \bar{\omega}=\bar{\varphi}, & s \bar{\varphi}=0,
\end{array}
$$

and

$$
s \phi=0,
$$


so that the operator $s$ is nilpotent

$$
s^{2}=0
$$

The fields $(\bar{\omega}, \omega)$ are anti-commuting and have ghost number $(-1,1)$, while $(\bar{\varphi}, \varphi)$ are a pair of complex conjugate commuting fields, carrying vanishing ghost number. For the BRST invariant action we write

$$
S_{\mathrm{inv}}=S_{\phi}+S_{\text {exact }}
$$

where

$$
\begin{aligned}
S_{\text {exact }} & =s \int d^{4} x\left(\bar{\omega}\left(-\partial^{2}\right) \varphi+\rho^{2} \bar{\omega} \varphi\right) \\
& =\int d^{4} x\left(\bar{\varphi}\left(-\partial^{2}\right) \varphi-\bar{\omega}\left(-\partial^{2}\right) \omega+\rho^{2}(\bar{\varphi} \varphi-\bar{\omega} \omega)\right) .
\end{aligned}
$$

The action $S_{\text {inv }}$ has the same physical content of $S_{\phi}$. This follows by observing that the integration over the fields $(\bar{\varphi}, \varphi, \bar{\omega}, \omega)$ amounts to introduce a unity factor in the path integral.

Further, we introduce a soft breaking of the BRST invariance, through the term

$$
S_{\vartheta}=\frac{\vartheta^{2}}{2} \int d^{4} x \phi(\varphi-\bar{\varphi})
$$

where $\vartheta$ is a mass parameter, here introduced by hand, which will enable us to modify the large distance behavior of the correlation functions $\left\langle\phi\left(x_{1}\right) \ldots \phi\left(x_{n}\right)\right\rangle$. Thus, for the starting action $S$, we have

$$
S=S_{\mathrm{inv}}+S_{\vartheta}
$$

and

$$
s S=\frac{\vartheta^{2}}{2} \int d^{4} x \phi \omega,
$$

showing that the BRST symmetry is softly broken. It is interesting to note that the fields $(\bar{\varphi}, \varphi, \bar{\omega}, \omega)$ can be integrated out also in the presence of the breaking term $S_{\vartheta}$, yielding the nonlocal action

$$
\int d^{4} x\left(\frac{1}{2} \phi\left(-\partial^{2}+\frac{\vartheta^{4}}{2} \frac{1}{\left(-\partial^{2}+\rho^{2}\right)}\right) \phi+\frac{\lambda}{4 !} \phi^{4}\right) .
$$

The fields $(\bar{\varphi}, \varphi, \bar{\omega}, \omega)$ can be seen thus as auxiliary fields needed to localize the term $\phi \frac{\vartheta^{4}}{\left(-\partial^{2}+\rho^{2}\right)} \phi$ in expression (17). In this sense, these fields play a role analogous to that of the auxiliary fields $\left(\bar{\varphi}_{\mu}^{a b}, \varphi_{\mu}^{a b}, \bar{\omega}_{\mu}^{a b}, \omega_{\mu}^{a b}\right)$ needed to localize the horizon function of the Gribov-Zwanziger action. In the same way, the parameter $\vartheta$ is akin to the Gribov parameter $\gamma$. Despite the presence of the soft breaking, eq.(16), the action $S$ turns out to be renormalizable. This is due to the fact that the $\operatorname{BRST}$ quartet $(\bar{\varphi}, \varphi, \bar{\omega}, \omega)$ is coupled in a linear way to the scalar field $\phi$, as exhibited by expression (14). As a consequence, the equations of motion of the fields $(\bar{\varphi}, \varphi, \bar{\omega}, \omega)$ acquire the meaning of linearly broken Ward identities, namely

$$
\begin{aligned}
& \frac{\delta S}{\delta \bar{\varphi}}=\left(-\partial^{2}+\rho^{2}\right) \varphi-\frac{\vartheta^{2}}{2} \phi, \\
& \frac{\delta S}{\delta \varphi}=\left(-\partial^{2}+\rho^{2}\right) \bar{\varphi}+\frac{\vartheta^{2}}{2} \phi, \\
& \frac{\delta S}{\delta \bar{\omega}}=-\left(-\partial^{2}+\rho^{2}\right) \omega, \\
& \frac{\delta S}{\delta \omega}=\left(-\partial^{2}+\rho^{2}\right) \bar{\omega} .
\end{aligned}
$$


These identities imply that the most general counterterm turns out to be independent from the fields of the BRST quartet. It depends only on the scalar field $\phi$, ensuring thus that the theory is renormalizable. In other words, the soft breaking of the BRST symmetry can be kept under control at the quantum level.

Let us give a look at the propagators:

$$
\begin{aligned}
\langle\phi(k) \phi(-k)\rangle & =\frac{k^{2}+\rho^{2}}{k^{4}+\rho^{2} k^{2}+\frac{\vartheta^{4}}{2}}, \\
\langle\phi(k) \varphi(-k)\rangle & =\frac{\vartheta^{2}}{2} \frac{1}{k^{4}+\rho^{2} k^{2}+\frac{\vartheta^{4}}{2}}, \\
\langle\phi(k) \bar{\varphi}(-k)\rangle & =-\frac{\vartheta^{2}}{2} \frac{1}{k^{4}+\rho^{2} k^{2}+\frac{\vartheta^{4}}{2}} .
\end{aligned}
$$

From expressions (19) it is apparent that the correlation functions of the scalar field, $\left\langle\phi\left(x_{1}\right) \ldots \phi\left(x_{n}\right)\right\rangle$, are modified in the infrared region, due to the presence of the soft parameters $(\vartheta, \rho)$. Moreover, one also observes that, in the absence of the BRST breaking, i.e. when $\vartheta=0$, the propagator of the scalar field $\phi$ turns out to be independent from the parameter $\rho$, which becomes an unphysical gauge parameter. As a consequence, in the absence of the breaking, the Green's functions $\left\langle\phi\left(x_{1}\right) \ldots \phi\left(x_{n}\right)\right\rangle$ will be independent from $\rho$ as well. However, for non-vanishing $\vartheta$, the parameter $\rho$ is no longer a gauge parameter, entering the expression of $\left\langle\phi\left(x_{1}\right) \ldots . \phi\left(x_{n}\right)\right\rangle$.

The framework outlined here gives us a way to introduce infrared effects in a local and renormalizable fashion. Nevertheless, the presence of the soft breaking of the BRST symmetry does not enable us to make use of the BRST operator $s$ in order to characterize the vacuum of the theory. Due to the lack of a conserved charge, one cannot impose that the vacuum is annihilated by the BRST operator. This means that the vacuum expectation value of quantities which are BRST exact does not necessarily vanish, i.e.

$$
\langle 0|s \Theta(x)| 0\rangle \neq 0
$$

So far, the only way to access correlation functions of the type of (20) is through explicit computations. Let us give an example by considering the dimension two condensate $\langle(\bar{\varphi}(x) \varphi(x)-\bar{\omega}(x) \omega(x))\rangle$, which has precisely the form of (20), i.e.

$$
\langle(\bar{\varphi}(x) \varphi(x)-\bar{\omega}(x) \omega(x))\rangle=\langle s(\bar{\omega}(x) \varphi(x))\rangle .
$$

This condensate can be seen as the analogue of the condensate $\left\langle 0\left|\left(\bar{\varphi}_{\mu}^{a b} \varphi_{\mu}^{a b}-\bar{\omega}_{\mu}^{a b} \omega_{\mu}^{a b}\right)\right| 0\right\rangle$ occurring in the case of the Gribov-Zwanziger theory. Expression (21) can be obtained by differentiating the vacuum energy $E_{v}$ with respect to the parameter $\rho$. In fact, from

$$
e^{-V E_{v}}=\int[D \Phi] e^{-S}
$$

it follows that

$$
\frac{\partial E_{v}}{\partial \rho^{2}}=\frac{\int[D \Phi](\bar{\varphi} \varphi-\bar{\omega} \omega) e^{-S}}{\int[D \Phi] e^{-S}}=\langle(\bar{\varphi} \varphi-\bar{\omega} \omega)\rangle
$$


Making use of the dimensional regularization, the explicit evaluation of the one-loop vacuum energy gives

$$
E_{v}=\frac{1}{2} \int \frac{d^{d} p}{(2 \pi)^{d}}\left(\log \left(p^{4}+p^{2} \rho^{2}+\frac{\vartheta^{4}}{2}\right)-\log \left(p^{2}+\rho^{2}\right)\right) .
$$

Thus,

$$
\langle(\bar{\varphi} \varphi-\bar{\omega} \omega)\rangle=\langle s(\bar{\omega} \varphi)\rangle=-\frac{\vartheta^{4}}{4} \int \frac{d^{4} p}{(2 \pi)^{4}} \frac{1}{\left(p^{4}+p^{2} \rho^{2}+\frac{\vartheta^{4}}{2}\right)\left(p^{2}+\rho^{2}\right)},
$$

where the integral in the right hand side of eq.(25) is convergent. Therefore, we see that the condensate $\langle(\bar{\varphi} \varphi-\bar{\omega} \omega)\rangle$ is non-vanishing for non-vanishing $\vartheta$. This example illustrates in a simple way that the we cannot access the vacuum state of the theory with the operator $s$.

From expression (25) one also sees that the condensate $\langle(\bar{\varphi} \varphi-\bar{\omega} \omega)\rangle$ is non-vanishing when the parameter $\rho$ is set to zero, i.e.

$$
\langle(\bar{\varphi} \varphi-\bar{\omega} \omega)\rangle_{\rho=0}=-\frac{\vartheta^{4}}{4} \int \frac{d^{4} p}{(2 \pi)^{4}} \frac{1}{\left(p^{4}+\frac{\vartheta^{4}}{2}\right) p^{2}} \neq 0 .
$$

Even if the operator $(\bar{\varphi} \varphi-\bar{\omega} \omega)$ were not included in the starting action, it would have been generated dynamically, as it is apparent from eq.(26). Therefore, the introduction of the parameter $\rho$ enables us to take into account the operator $(\bar{\varphi} \varphi-\bar{\omega} \omega)$ from the beginning. As we shall see, a similar situation will be encountered in the case of the Gribov-Zwanziger action.

\subsection{The modified BRST operator}

Let us look now at the possibility of finding a modified BRST operator which corresponds to an exact invariance of the action $S$. We shall rely on the property that the breaking term, eq.(16), is quadratic in the fields, due to the fact that the BRST quartet couples linearly to the scalar field $\phi$, see expression (14). Let us consider the equation of motion of the field $\bar{\omega}$, i.e.

$$
\frac{\delta S}{\delta \bar{\omega}(x)}=-\left(-\partial^{2}+\rho^{2}\right) \omega(x)
$$

We observe that the operator $\left(-\partial^{2}+\rho^{2}\right)$ is positive definite, so that its inverse does exist. As a consequence, eq.(27) can be solved for $\omega(x)$, yielding

$$
\omega(x)=-\frac{1}{\left(-\partial^{2}+\rho^{2}\right)} \frac{\delta S}{\delta \bar{\omega}} \equiv-\int d^{4} y \frac{1}{\left(-\partial^{2}+\rho^{2}\right)_{x y}} \frac{\delta S}{\delta \bar{\omega}(y)},
$$

where

$$
\frac{1}{\left(-\partial^{2}+\rho^{2}\right)_{x y}}=\int \frac{d^{4} p}{(2 \pi)^{4}} \frac{1}{p^{2}+\rho^{2}} e^{-i p(x-y)} .
$$

Therefore, equation (16) can be rewritten as

$$
s S=-\frac{\vartheta^{2}}{2} \int d^{4} x \phi \frac{1}{\left(-\partial^{2}+\rho^{2}\right)} \frac{\delta S}{\delta \bar{\omega}},
$$


showing that the BRST breaking of eq.(16) can be cast in the form of a contact term, i.e. of a term related to the equations of motion. Equation (30) implies that the action $S$ is left invariant by the modified nilpotent operator $s_{\vartheta}$

$$
\begin{aligned}
s_{\vartheta} \bar{\omega} & =\bar{\varphi}+\frac{\vartheta^{2}}{2} \frac{1}{\left(-\partial^{2}+\rho^{2}\right)} \phi, \\
s_{\vartheta} \bar{\varphi} & =0, \\
s_{\vartheta} \varphi & =\omega, \\
s_{\vartheta} \omega & =0, \\
s_{\vartheta} \phi & =0,
\end{aligned}
$$

so that

$$
s_{\vartheta} S=0 .
$$

The operator $s_{\vartheta}$ exhibits explicit dependence from the parameter $\vartheta$. Moreover, it reduces to the operator $s$ when $\vartheta=0$. One should notice, however, that the operator $s_{\vartheta}$ is non-local. As such, it cannot be used to analyse the renormalizability properties of the model. Nevertheless, its existence is helpful in order to evaluate the expectation value of BRST exact quantities, by requiring that

$$
\left\langle 0\left|s_{\vartheta} \Theta(x)\right| 0\right\rangle=0
$$

To give an example of the usefulness of eq.(33), let us compute again the condensate $\langle(\bar{\varphi} \varphi-\bar{\omega} \omega)\rangle$, by making use of condition (33). From the equation

$$
\left\langle s_{\vartheta}(\bar{\omega} \varphi)\right\rangle=0
$$

it follows that

$$
\langle(\bar{\varphi} \varphi-\bar{\omega} \omega)\rangle+\frac{\vartheta^{2}}{2}\left\langle\phi \frac{1}{\left(-\partial^{2}+\rho^{2}\right)} \varphi\right\rangle=0
$$

Thus

$$
\begin{aligned}
\langle s(\bar{\omega} \varphi)\rangle & =\langle(\bar{\varphi} \varphi-\bar{\omega} \omega)\rangle=-\frac{\vartheta^{2}}{2}\left\langle\phi \frac{1}{\left(-\partial^{2}+\rho^{2}\right)} \varphi\right\rangle \\
& =-\frac{\vartheta^{2}}{2} \int d^{4} y \frac{1}{\left(-\partial^{2}+\rho^{2}\right)_{x y}} \int \frac{d^{4} q}{(2 \pi)^{4}}\langle\phi(q) \varphi(-q)\rangle e^{-i q(x-y)} \\
& =-\frac{\vartheta^{2}}{2} \int d^{4} y \frac{d^{4} q}{(2 \pi)^{4}} \frac{d^{4} p}{(2 \pi)^{4}} \frac{1}{p^{2}+\rho^{2}}\langle\phi(q) \varphi(-q)\rangle e^{-i q(x-y)} e^{-i p(x-y)} \\
& =-\frac{\vartheta^{2}}{2} \int \frac{d^{4} q}{(2 \pi)^{4}} \frac{1}{q^{2}+\rho^{2}}\langle\phi(q) \varphi(-q)\rangle .
\end{aligned}
$$

Finally, from equations (19) we obtain

$$
\langle s(\bar{\omega} \varphi)\rangle=\langle(\bar{\varphi} \varphi-\bar{\omega} \omega)\rangle=-\frac{\vartheta^{4}}{4} \int \frac{d^{4} p}{(2 \pi)^{4}} \frac{1}{\left(p^{4}+p^{2} \rho^{2}+\frac{\vartheta^{4}}{2}\right)\left(p^{2}+\rho^{2}\right)},
$$

in agreement with expression (25). We see thus that condition (33) gives us a practical way of evaluating correlators of the kind $\langle 0|s \Theta(x)| 0\rangle$. 


\subsection{The Noether current}

Let us conclude the analysis of the toy model by deriving the Noether current corresponding to the exact invariance (32). To that purpose, we make use of the functional form of the operator $s_{\vartheta}$, i.e.

$$
\begin{aligned}
s_{\vartheta} & =\int d^{4} x w(x) \\
w(x) & =\left(\bar{\varphi}(x)+\frac{\vartheta^{2}}{2} \Lambda(x)\right) \frac{\delta}{\delta \bar{\omega}(x)}+\omega(x) \frac{\delta}{\delta \varphi(x)},
\end{aligned}
$$

where $\Lambda(x)$ stands for

$$
\Lambda(x)=\int d^{4} y \frac{1}{\left(-\partial^{2}+\rho^{2}\right)_{x y}} \phi(y)=\int d^{4} y \frac{d^{4} p}{(2 \pi)^{4}} \frac{1}{p^{2}+\rho^{2}} e^{-i p(x-y)} \phi(y)
$$

so that

$$
\left(-\partial_{x}^{2}+\rho^{2}\right) \Lambda(x)=\phi(x) .
$$

Acting with the operator $w(x)$ on the action $S$ one finds

$$
\begin{aligned}
w(x) S & =\left(\bar{\varphi}(x)+\frac{\vartheta^{2}}{2} \Lambda(x)\right)\left(\partial^{2} \omega-\rho^{2} \omega\right)+\left(-\partial^{2} \bar{\varphi}+\rho^{2} \bar{\varphi}\right) \omega+\frac{\vartheta^{2}}{2} \phi \omega \\
& =\bar{\varphi} \partial^{2} \omega-\left(\partial^{2} \bar{\varphi}\right) \omega+\frac{\vartheta^{2}}{2}\left(\Lambda \partial^{2} \omega-\rho^{2} \Lambda \omega\right)+\frac{\vartheta^{2}}{2} \phi \omega \\
& =\partial_{\mu}\left(\bar{\varphi} \partial_{\mu} \omega-\left(\partial_{\mu} \bar{\varphi}\right) \omega\right)+\frac{\vartheta^{2}}{2}\left(\partial_{\mu}\left(\Lambda \partial_{\mu} \omega-\left(\partial_{\mu} \Lambda\right) \omega\right)+\left(\left(\partial^{2}-\rho^{2}\right) \Lambda\right) \omega\right)+\frac{\vartheta^{2}}{2} \phi \omega
\end{aligned}
$$

Making use of eq.(40), it follows

$$
w(x) S=\partial_{\mu} j_{\mu}
$$

where the Noether current $j_{\mu}$ is given by

$$
j_{\mu}=\left(\bar{\varphi} \partial_{\mu} \omega-\left(\partial_{\mu} \bar{\varphi}\right) \omega\right)+\frac{\vartheta^{2}}{2}\left(\Lambda \partial_{\mu} \omega-\left(\partial_{\mu} \Lambda\right) \omega\right)
$$

\subsection{Preliminary considerations}

Several remarks can be made from the example considered here.

- The mechanism of the soft breaking of the BRST enables us to modify in a smooth way the correlation functions of the theory in the infrared, due to the presence of the soft parameters $(\vartheta, \rho)$.

- The introduction of the BRST quartet and of the corresponding breaking term are done in a way which preserves locality and renormalizability. 
- However, due to the presence of the breaking, the BRST operator $s$ does not allow us to characterize the vacuum of the theory. Nevertheless, it turns out that the action $S$ is left invariant by a modified operator $s_{\vartheta}$, which exhibits explicit dependence from the parameter $\vartheta$, while reducing to the BRST operator $s$ for vanishing $\vartheta$. Although non-local, the new operator $s_{\vartheta}$ can be employed to obtain the explicit expression of quantities which are BRST exact.

\section{The Gribov-Zwanziger action}

\subsection{The non-local horizon function and its localization}

Let us start by giving a short overview of the Gribov-Zwanziger framework, enabling us to implement the restriction in the Euclidean functional integral to the Gribov region $\Omega$ [3], defined as the set of field configurations fulfilling the Landau gauge condition and for which the Faddeev-Popov operator,

$$
\mathcal{M}^{a b}=-\partial_{\mu}\left(\partial_{\mu} \delta^{a b}+g f^{a c b} A_{\mu}^{c}\right),
$$

is strictly positive, namely

$$
\Omega \equiv\left\{A_{\mu}^{a}, \partial_{\mu} A_{\mu}^{a}=0, \mathcal{M}^{a b}>0\right\} .
$$

This amounts to adding to the Yang-Mills action

$$
S_{\mathrm{YM}}=\frac{1}{4} \int d^{4} x F_{\mu \nu}^{a} F_{\mu \nu}^{a},
$$

the horizon term [6, 7]

$$
\begin{aligned}
S_{h} & =\gamma^{4} \int d^{4} x h(x), \\
h(x) & =g^{2} f^{a b c} A_{\mu}^{b}\left(\mathcal{M}^{-1}\right)^{a d} f^{d e c} A_{\mu}^{e},
\end{aligned}
$$

where the non-local kernel $\left(\mathcal{M}^{-1}\right)^{a d}$ stands for the inverse of the Faddeev-Popov operator

$$
\mathcal{M}^{a b}(x)\left[\left(\mathcal{M}^{-1}(x, y)\right)^{b c}\right]=\delta^{4}(x-y) \delta^{a c} .
$$

Thus, for the partition function implementing the restriction to $\Omega$ one has [6, 7 ]

$$
\int_{\Omega} D A \delta(\partial A) \operatorname{det} \mathcal{M} e^{-S_{Y M}}=\int D A \delta(\partial A) \operatorname{det} \mathcal{M} e^{-\left(S_{Y M}+S_{h}\right)}
$$

where the Gribov parameter $\gamma$ is determined by the horizon condition [6, 7 ]

$$
\langle h(x)\rangle=4\left(N^{2}-1\right) .
$$

As already mentioned, the non-local horizon function can be localized through the introduction of a suitable set of additional fields [6, 7]

$$
e^{-S_{h}}=\int D \varphi D \bar{\varphi} D \omega D \bar{\omega} e^{-S_{\mathrm{loc}}}
$$


with

$$
S_{\mathrm{loc}}=\int d^{4} x\left(-\bar{\varphi}_{\mu}^{a c} \mathcal{M}^{a b} \varphi_{\mu}^{b c}+\bar{\omega}_{\mu}^{a c} \mathcal{M}^{a b} \omega_{\mu}^{b c}\right)-\gamma^{2} g \int d^{4} x\left(f^{a b c} A_{\mu}^{a} \varphi_{\mu}^{b c}+f^{a b c} A_{\mu}^{a} \bar{\varphi}_{\mu}^{b c}\right)
$$

The fields $\left(\bar{\varphi}_{\mu}^{a c}, \varphi_{\mu}^{a c}\right)$ are a pair of complex conjugate bosonic fields, while $\left(\bar{\omega}_{\mu}^{a c}, \omega_{\mu}^{a c}\right)$ are anticommuting fields. Each of these fields has $4\left(N^{2}-1\right)^{2}$ components. Therefore, a local action is obtained from the restriction to the Gribov region, namely

$$
\int_{\Omega} D A \delta(\partial A) \operatorname{det} \mathcal{M} e^{-S_{Y M}}=\int D A D \varphi D \bar{\varphi} D \omega D \bar{\omega} D b D c D \bar{c} e^{-S_{\mathrm{GZ}}}
$$

where $S_{\mathrm{GZ}}$ is the Gribov-Zwanziger action, given by

$$
\begin{aligned}
S_{\mathrm{GZ}}= & S_{\mathrm{YM}}+\int d^{4} x\left(b^{a} \partial_{\mu} A_{\mu}^{a}-\bar{c}^{a} \mathcal{M}^{a b} c^{b}\right)+\int d^{4} x\left(-\bar{\varphi}_{\mu}^{a c} \mathcal{M}^{a b} \varphi_{\mu}^{b c}+\bar{\omega}_{\mu}^{a c} \mathcal{M}^{a b} \omega_{\mu}^{b c}\right) \\
& -\gamma^{2} g \int d^{4} x\left(f^{a b c} A_{\mu}^{a} \varphi_{\mu}^{b c}+f^{a b c} A_{\mu}^{a} \bar{\varphi}_{\mu}^{b c}+\frac{4}{g}\left(N^{2}-1\right) \gamma^{2}\right) .
\end{aligned}
$$

The constant term, $4\left(N^{2}-1\right) \gamma^{4}$, in expression (54) is introduced in order to rewrite the horizon condition (150) as

$$
\frac{\partial \Gamma}{\partial \gamma^{2}}=0
$$

with $\Gamma$ being the effective action obtained from $S_{\mathrm{GZ}}$

$$
\mathrm{e}^{-\Gamma}=\int[D \Phi] \mathrm{e}^{-S_{\mathrm{GZ}}}
$$

To the first order, the gap equation (55) takes the form

$$
1=\frac{3}{4} N g^{2} \int \frac{d^{4} p}{(2 \pi)^{4}} \frac{1}{p^{4}+2 g^{2} N \gamma^{4}} .
$$

Let us also observe that condition (55) is equivalent to

$$
\left\langle 0\left|g f^{a b c} A_{\mu}^{a} \varphi_{\mu}^{b c}\right| 0\right\rangle+\left\langle 0\left|g f^{a b c} A_{\mu}^{a} \bar{\varphi}_{\mu}^{b c}\right| 0\right\rangle=-8\left(N^{2}-1\right) \gamma^{2}
$$

which, owing to the discrete symmetry of the action $S_{\mathrm{GZ}}$

$$
\begin{aligned}
\bar{\varphi}_{\mu}^{b c} & \rightarrow \varphi_{\mu}^{b c} \\
\varphi_{\mu}^{b c} & \rightarrow \bar{\varphi}_{\mu}^{b c}, \\
b^{a} & \rightarrow\left(b^{a}-g f^{a m n} \bar{\varphi}_{\mu}^{m c} \varphi_{\mu}^{n c}\right)
\end{aligned}
$$

becomes

$$
\left\langle 0\left|g f^{a b c} A_{\mu}^{a} \varphi_{\mu}^{b c}\right| 0\right\rangle=\left\langle 0\left|g f^{a b c} A_{\mu}^{a} \bar{\varphi}_{\mu}^{b c}\right| 0\right\rangle=-4\left(N^{2}-1\right) \gamma^{2}=-\gamma^{2}\langle h(x)\rangle
$$

Equation (60) shows the relevance of the dimension two condensates $\left\langle 0\left|g f^{a b c} A_{\mu}^{a} \varphi_{\mu}^{b c}\right| 0\right\rangle,\left\langle 0\left|g f^{a b c} A_{\mu}^{a} \bar{\varphi}_{\mu}^{b c}\right| 0\right\rangle$, within the Gribov-Zwanziger approach. 


\subsection{Soft breaking of the BRST invariance}

As already underlined, the auxiliary fields $\left(\bar{\varphi}_{\mu}^{a b}, \varphi_{\mu}^{a b}, \bar{\omega}_{\mu}^{a b}, \omega_{\mu}^{a b}\right)$ needed to localize the horizon function (47) give rise to a BRST quartet, meaning that they are assembled in two BRST doublets [6, 7], see eqs.(3). Acting thus with the BRST operator $s$ on the action (54), one finds

$$
\begin{aligned}
s S_{\mathrm{GZ}}= & \int d^{4} x g f^{a b m}\left(D_{\nu}^{b p} c^{p}\right)\left(\left(\partial_{\nu} \bar{\varphi}_{\mu}^{a c}\right) \varphi_{\mu}^{m c}-\left(\partial_{\nu} \bar{\omega}_{\mu}^{a c}\right) \omega_{\mu}^{m c}\right) \\
& -\gamma^{2} g \int d^{4} x\left(c^{m} D_{\mu}^{m a}\left(f^{a b c}\left(\varphi_{\mu}^{b c}+\bar{\varphi}_{\mu}^{b c}\right)\right)+f^{a b c} A_{\mu}^{a} \omega_{\mu}^{b c}\right),
\end{aligned}
$$

which shows that the BRST symmetry is broken. One notes that the first term in the right-hand side of expression (61) can be written as the BRST variation of $g f^{a b m}\left(D_{\nu}^{b p} c^{p}\right)\left(\partial_{\nu} \bar{\omega}_{\mu}^{a c}\right) \varphi_{\mu}^{m c}$, i.e.

$$
g f^{a b m}\left(D_{\nu}^{b p} c^{p}\right)\left(\left(\partial_{\nu} \bar{\varphi}_{\mu}^{a c}\right) \varphi_{\mu}^{m c}-\left(\partial_{\nu} \bar{\omega}_{\mu}^{a c}\right) \omega_{\mu}^{m c}\right)=-s\left(g f^{a b m}\left(D_{\nu}^{b p} c^{p}\right)\left(\partial_{\nu} \bar{\omega}_{\mu}^{a c}\right) \varphi_{\mu}^{m c}\right),
$$

so that it does not correspond to a real breaking. The terms which give rise to the true BRST soft breaking are those proportional to the Gribov parameter $\gamma$. The expression $g f^{a b m}\left(D_{\nu}^{b p} c^{p}\right)\left(\partial_{\nu} \bar{\omega}_{\mu}^{a c}\right) \varphi_{\mu}^{m c}$ is usually introduced in the starting Gribov-Zwanziger action. It can be obtained through a change of variables [6, 7] in expression (53), the corresponding Jacobian being field independent. However, for the purposes of the present work, we keep the term $g f^{a b m}\left(D_{\nu}^{b p} c^{p}\right)\left(\left(\partial_{\nu} \bar{\varphi}_{\mu}^{a c}\right) \varphi_{\mu}^{m c}-\left(\partial_{\nu} \bar{\omega}_{\mu}^{a c}\right) \omega_{\mu}^{m c}\right)$ as it stands.

\subsection{The operator $s_{\gamma}$}

To construct the generalized operator $s_{\gamma}$, we proceed as in the toy model considered in the previous section. From the Gribov-Zwanziger action, we get

$$
\begin{aligned}
& \frac{\delta S_{\mathrm{GZ}}}{\delta \bar{c}^{a}}=-\mathcal{M}^{a b} c^{b}, \\
& \frac{\delta S_{\mathrm{GZ}}}{\delta \bar{\omega}_{\mu}^{a c}}=\mathcal{M}^{a b} \omega_{\mu}^{b c} .
\end{aligned}
$$

We observe now that, within the Gribov region $\Omega$, eq.(45), the Faddeev-Popov operator $\mathcal{M}^{a b}$ is strictly positive, so that its inverse $\left(\mathcal{M}^{-1}\right)^{a b}$ does exist. This property enables us to solve equations (63) for the fields $c^{b}$ and $\omega_{\mu}^{b c}$, obtaining

$$
\begin{aligned}
c^{b}(x) & =-\left(\mathcal{M}^{-1}\right)_{x y}^{b c} \frac{\delta S_{\mathrm{GZ}}}{\delta \bar{c}_{y}^{c}}=-\int d^{4} y\left[\left(\mathcal{M}^{-1}(x, y)\right)^{b c}\right] \frac{\delta S_{\mathrm{GZ}}}{\delta \bar{c}^{c}(y)}, \\
\omega_{\mu}^{d c}(x) & =\left(\mathcal{M}^{-1}\right)_{x y}^{d a} \frac{\delta S_{\mathrm{GZ}}}{\delta \bar{\omega}_{y \mu}^{a c}}=\int d^{4} y\left[\left(\mathcal{M}^{-1}(x, y)\right)^{d a}\right] \frac{\delta S_{\mathrm{GZ}}}{\delta \bar{\omega}^{a c}(y)}
\end{aligned}
$$

Thus, equation (61) can be rewritten as

$$
s S_{\mathrm{GZ}}=\int d^{4} x\left(-\left(D_{\nu}^{m a} \Lambda_{\nu}^{a}\right)_{x}\left(\mathcal{M}^{-1}\right)_{x y}^{m c} \frac{\delta S_{\mathrm{GZ}}}{\delta \bar{c}_{y}^{c}}-\gamma^{2} g f^{a b c} A_{\mu}^{a}(x)\left(\mathcal{M}^{-1}\right)_{x y}^{b m} \frac{\delta S_{\mathrm{GZ}}}{\delta \bar{\omega}_{y \mu}^{m c}}\right)
$$


where $\Lambda_{\nu}^{a}$ stands for

$$
\Lambda_{\nu}^{a}=-\gamma^{2} g f^{a b c}\left(\varphi_{\nu}^{b c}+\bar{\varphi}_{\nu}^{b c}\right)-g f^{b a p}\left(\left(\partial_{\nu} \bar{\varphi}_{\mu}^{b c}\right) \varphi_{\mu}^{p c}-\left(\partial_{\nu} \bar{\omega}_{\mu}^{b c}\right) \omega_{\mu}^{p c}\right)
$$

As in the case of the toy model, we see that the soft breaking of the BRST symmetry turns out to be related to contact terms, enabling us to introduce the extended operator we are looking for. Eq.(65) expresses precisely the exact invariance of the Gribov-Zwanziger action $S_{\mathrm{GZ}}$ under the operator $s_{\gamma}$

$$
s_{\gamma} S_{\mathrm{GZ}}=0
$$

where $s_{\gamma}$ is given by

$$
\begin{aligned}
s_{\gamma} A_{\mu}^{a} & =-\left(D_{\mu} c\right)^{a} \\
s_{\gamma} c^{a} & =\frac{1}{2} g f^{a b c} c^{b} c^{c} \\
s_{\gamma} \bar{c}^{a}(x) & =b^{a}(x)+\int d^{4} y\left(D_{\nu}^{m c} \Lambda_{\nu}^{c}\right)_{y}\left(\mathcal{M}^{-1}\right)_{y x}^{m a}, \\
s_{\gamma} b^{a} & =0 \\
s_{\gamma} \bar{\omega}_{\mu}^{a b}(x) & =\bar{\varphi}_{\mu}^{a b}(x)+\gamma^{2} g \int d^{4} y f^{m p b} A_{\mu}^{m}(y)\left(\mathcal{M}^{-1}\right)_{y x}^{p a} \\
s_{\gamma} \bar{\varphi}_{\mu}^{a b} & =0 \\
s_{\gamma} \varphi_{\mu}^{a b} & =\omega_{\mu}^{a b}, \\
s_{\gamma} \omega_{\mu}^{a b} & =0 .
\end{aligned}
$$

Like the operator $s_{\vartheta}$ of eq.(31), the modified operator $s_{\gamma}$ is non-local. However, unlike $s_{\vartheta}$, it lacks nilpotency. Nevertheless, it turns out to be helpful in order to evaluate the vacuum expectation value of BRST exact quantities, like the condensate $\left\langle 0\left|\left(\bar{\varphi}_{\mu}^{a b}(x) \varphi_{\mu}^{a b}(x)-\bar{\omega}_{\mu}^{a b}(x) \omega_{\mu}^{a b}(x)\right)\right| 0\right\rangle$.

\subsection{Evaluation of $\left\langle 0\left|\left(\bar{\varphi}_{\mu}^{a b}(x) \varphi_{\mu}^{a b}(x)-\bar{\omega}_{\mu}^{a b}(x) \omega_{\mu}^{a b}(x)\right)\right| 0\right\rangle$}

To evaluate the condensate $\left\langle 0\left|\left(\bar{\varphi}_{\mu}^{a b}(x) \varphi_{\mu}^{a b}(x)-\bar{\omega}_{\mu}^{a b}(x) \omega_{\mu}^{a b}(x)\right)\right| 0\right\rangle$ we start from the condition

$$
\left\langle 0\left|s_{\gamma}\left(\bar{\omega}_{\mu}^{a b}(x) \varphi_{\mu}^{a b}(x)\right)\right| 0\right\rangle=0 .
$$

Making use of transformations (68), we get

$$
\left\langle 0\left|s_{\gamma}\left(\bar{\omega}_{\mu}^{a b}(x) \varphi_{\mu}^{a b}(x)\right)\right| 0\right\rangle=\left\langle 0\left|\left(\bar{\varphi}_{\mu}^{a b} \varphi_{\mu}^{a b}-\bar{\omega}_{\mu}^{a b} \omega_{\mu}^{a b}\right)\right| 0\right\rangle+\gamma^{2} g f^{a b c} \int d^{4} y\left\langle 0\left|A_{\mu}^{a}(y)\left(\mathcal{M}^{-1}\right)_{y x}^{b m} \varphi_{\mu}^{m c}(x)\right| 0\right\rangle
$$

Therefore

$$
\left\langle 0\left|\left(\bar{\varphi}_{\mu}^{a b}(x) \varphi_{\mu}^{a b}(x)-\bar{\omega}_{\mu}^{a b}(x) \omega_{\mu}^{a b}(x)\right)\right| 0\right\rangle=-\gamma^{2} g f^{a b c} \int d^{4} y\left\langle 0\left|A_{\mu}^{a}(y)\left(\mathcal{M}^{-1}\right)_{y x}^{b m} \varphi_{\mu}^{m c}(x)\right| 0\right\rangle .
$$

Equation (71) is a non-perturbative Ward identity relating the dimension two condensate to the Green's function appearing in the right-hand side. One should also notice that expression (71) displays explicit dependence from the Gribov parameter $\gamma$, as expected from the non-perturbative 
nature of $\left\langle 0\left|\left(\bar{\varphi}_{\mu}^{a b} \varphi_{\mu}^{a b}-\bar{\omega}_{\mu}^{a b} \omega_{\mu}^{a b}\right)\right| 0\right\rangle$.

To the first order approximation

$$
\left(\mathcal{M}^{-1}\right)_{y x}^{b m}=\left(\frac{1}{-\partial^{2}}\right)_{y x} \delta^{b m}+\ldots
$$

so that

$$
\begin{aligned}
\left\langle 0\left|\left(\bar{\varphi}_{\mu}^{a b} \varphi_{\mu}^{a b}-\bar{\omega}_{\mu}^{a b} \omega_{\mu}^{a b}\right)\right| 0\right\rangle & =-\gamma^{2} g f^{a b c} \int d^{4} y\left\langle 0\left|A_{\mu}^{a}(y)\left(\frac{1}{-\partial^{2}}\right)_{y x} \varphi_{\mu}^{b c}(x)\right| 0\right\rangle \\
& =-\gamma^{2} g f^{a b c} \int d^{4} y \int \frac{d^{4} p}{(2 \pi)^{4}} \frac{1}{p^{2}} e^{-i p(x-y)}\left\langle 0\left|A_{\mu}^{a}(y) \varphi_{\mu}^{b c}(x)\right| 0\right\rangle \\
& =-\gamma^{2} g f^{a b c} \int \frac{d^{4} p}{(2 \pi)^{4}} \frac{1}{p^{2}}\left\langle 0\left|A_{\mu}^{a}(p) \varphi_{\mu}^{b c}(-p)\right| 0\right\rangle .
\end{aligned}
$$

For the propagator $\left\langle 0\left|A_{\mu}^{a}(p) \varphi_{\nu}^{b c}(-p)\right| 0\right\rangle$, we have [1]

$$
\begin{aligned}
\left\langle 0\left|A_{\mu}^{a}(p) \varphi_{\nu}^{b c}(-p)\right| 0\right\rangle & =-\frac{f^{a b c} \widehat{\gamma}^{2}}{\sqrt{2}\left(p^{4}+N \widehat{\gamma}^{4}\right)}\left(\delta_{\mu \nu}-\frac{p_{\mu} p_{\nu}}{p^{2}}\right), \\
\widehat{\gamma}^{2} & =\sqrt{2} g \gamma^{2} .
\end{aligned}
$$

Therefore

$$
\begin{aligned}
\left\langle 0\left|\left(\bar{\varphi}_{\mu}^{a b} \varphi_{\mu}^{a b}-\bar{\omega}_{\mu}^{a b} \omega_{\mu}^{a b}\right)\right| 0\right\rangle & =\frac{3}{\sqrt{2}} \gamma^{2} g f^{a b c} f^{a b c} \widehat{\gamma}^{2} \int \frac{d^{4} p}{(2 \pi)^{4}} \frac{1}{p^{2}} \frac{1}{\left(p^{4}+N \widehat{\gamma}^{4}\right)} \\
& =\frac{3 \sqrt{2}}{64 \pi} g \sqrt{N}\left(N^{2}-1\right) \gamma^{2},
\end{aligned}
$$

where use has been made of

$$
f^{a b c} f^{d b c}=N \delta^{a d} .
$$

Expression (75) agrees with the result already found in [11].

\subsection{Remarks on the condition $\left\langle 0\left|s_{\gamma} \Theta(x)\right| 0\right\rangle=0$}

In this section we aim at providing a better understanding of the condition $\left\langle 0\left|s_{\gamma} \Theta(x)\right| 0\right\rangle=0$. To that purpose, we shall study the quantity $\left\langle 0\left|s_{\gamma}\left(g f^{a b c} A_{\mu}^{a}(x) \bar{\omega}_{\mu}^{b c}(x)\right)\right| 0\right\rangle$.

From eqs.(68), we have

$$
\begin{aligned}
\left\langle 0\left|s_{\gamma}\left(g f^{a b c} A_{\mu}^{a} \bar{\omega}_{\mu}^{b c}\right)\right| 0\right\rangle= & -\left\langle 0\left|\left(g f^{a b c}\left(D_{\mu}^{a p} c^{p}\right) \bar{\omega}_{\mu}^{b c}\right)\right| 0\right\rangle+\left\langle 0\left|g f^{a b c} A_{\mu}^{a} \bar{\varphi}_{\mu}^{b c}\right| 0\right\rangle \\
& +\gamma^{2} g^{2} f^{a b c} \int d^{4} y f^{m p c}\left\langle 0\left|A_{\mu}^{a}(x) A_{\mu}^{m}(y)\left(\mathcal{M}^{-1}\right)_{y x}^{p b}\right| 0\right\rangle .
\end{aligned}
$$

Taking into account that expression $\left\langle 0\left|\left(g f^{a b c}\left(D_{\mu}^{a p} c^{p}\right) \bar{\omega}_{\mu}^{b c}\right)\right| 0\right\rangle$ vanishes, due to the discrete symmetry $\left(\bar{\omega}_{\mu}^{b c} \rightarrow-\bar{\omega}_{\mu}^{b c}, \omega_{\mu}^{b c} \rightarrow-\omega_{\mu}^{b c}\right)$ of the action (54), one gets

$$
\left\langle 0\left|s_{\gamma}\left(g f^{a b c} A_{\mu}^{a} \bar{\omega}_{\mu}^{b c}\right)\right| 0\right\rangle=\left\langle 0\left|g f^{a b c} A_{\mu}^{a} \bar{\varphi}_{\mu}^{b c}\right| 0\right\rangle+\gamma^{2} g^{2} f^{a b c} \int d^{4} y f^{m p c}\left\langle 0\left|A_{\mu}^{a}(x) A_{\mu}^{m}(y)\left(\mathcal{M}^{-1}\right)_{y x}^{p b}\right| 0\right\rangle .
$$


Reminding now the expression of the horizon function $h(x)$ of eq.(47), it follows

$$
\left\langle 0\left|s_{\gamma}\left(g f^{a b c} A_{\mu}^{a} \bar{\omega}_{\mu}^{b c}\right)\right| 0\right\rangle=\left\langle 0\left|g f^{a b c} A_{\mu}^{a} \bar{\varphi}_{\mu}^{b c}\right| 0\right\rangle+\gamma^{2}\langle 0|h(x)| 0\rangle=0,
$$

due to equation (60).

We see thus that the equation $\left\langle 0\left|s_{\gamma}\left(g f^{a b c} A_{\mu}^{a} \bar{\omega}_{\mu}^{b c}\right)\right| 0\right\rangle=0$ follows from the gap equation (60) for the Gribov parameter $\gamma$. This provides support to the condition $\left\langle 0\left|s_{\gamma} \Theta(x)\right| 0\right\rangle=0$, which seems to emerge in a natural way within the Gribov-Zwanziger framework.

\subsection{A general formula}

We can now establish a general procedure to evaluate the BRST exact quantity $\langle 0|s \Theta(x)| 0\rangle$. Let us decompose the operator $s_{\gamma}$ as

$$
s_{\gamma}=s+\delta_{\gamma},
$$

where $s$ is the BRST operator of eqs.(3) and $\delta_{\gamma}$ stands for the operator

$$
\begin{aligned}
\delta_{\gamma} A_{\mu}^{a} & =0 \\
\delta_{\gamma} c^{a} & =0 \\
\delta_{\gamma} \bar{c}^{a}(x) & =\int d^{4} y\left(D_{\nu}^{m c} \Lambda_{\nu}^{c}\right)_{y}\left(\mathcal{M}^{-1}\right)_{y x}^{m a}, \\
\delta_{\gamma} b^{a} & =0 \\
\delta_{\gamma} \bar{\omega}_{\mu}^{a b}(x) & =\gamma^{2} g \int d^{4} y f^{m p b} A_{\mu}^{m}(y)\left(\mathcal{M}^{-1}\right)_{y x}^{p a} \\
\delta_{\gamma} \bar{\varphi}_{\mu}^{a b} & =0, \\
\delta_{\gamma} \varphi_{\mu}^{a b} & =0, \\
\delta_{\gamma} \omega_{\mu}^{a b} & =0 .
\end{aligned}
$$

Therefore, from the condition

$$
\left\langle 0\left|s_{\gamma} \Theta(x)\right| 0\right\rangle=0
$$

we get

$$
\langle 0|s \Theta(x)| 0\rangle=-\left\langle 0\left|\delta_{\gamma} \Theta(x)\right| 0\right\rangle .
$$

We see thus that the characterization of quantities which are BRST exact relies on the operator $\delta_{\gamma}$ which takes into account the presence of the Gribov horizon through its explicit dependence from the parameter $\gamma$.

\subsubsection{From condensates to correlation functions}

The formula (83) enables us to extend the previous construction to the case of correlation functions which are BRST exact. As an example, let us evaluate the two-point correlation function

$$
\left\langle\left(\bar{\varphi}_{\mu}^{a b}(x) \varphi_{\mu}^{a b}(y)-\bar{\omega}_{\mu}^{a b}(x) \omega_{\mu}^{a b}(y)\right)\right\rangle=\left\langle s\left(\bar{\omega}_{\mu}^{a b}(x) \varphi_{\mu}^{a b}(y)\right)\right\rangle .
$$


From the condition

$$
\left\langle s_{\gamma}\left(\bar{\omega}_{\mu}^{a b}(x) \varphi_{\mu}^{a b}(y)\right)\right\rangle=0,
$$

we easily get

$$
\left\langle 0\left|\left(\bar{\varphi}_{\mu}^{a b}(x) \varphi_{\mu}^{a b}(y)-\bar{\omega}_{\mu}^{a b}(x) \omega_{\mu}^{a b}(y)\right)\right| 0\right\rangle=-\gamma^{2} g f^{m p b} \int d^{4} z\left\langle 0\left|A_{\mu}^{m}(z)\left(\mathcal{M}^{-1}\right)_{z x}^{p a} \varphi_{\mu}^{m c}(y)\right| 0\right\rangle .
$$

\section{The refined Gribov-Zwanziger action}

The so called refined Gribov-Zwanziger action was introduced in [1, 11]. It takes into account the existence of the dimension two condensate $\left\langle 0\left|\left(\bar{\varphi}_{\mu}^{a b} \varphi_{\mu}^{a b}-\bar{\omega}_{\mu}^{a b} \omega_{\mu}^{a b}\right)\right| 0\right\rangle$, which is non-vanishing for non-vanishing Gribov parameter $\gamma$. This condensate reflects the non-trivial dynamics developed by the auxiliary fields $\left(\bar{\varphi}_{\mu}^{a b}, \varphi_{\mu}^{a b}, \bar{\omega}_{\mu}^{a b}, \omega_{\mu}^{a b}\right)$. This feature has motivated the introduction of the refined Gribov-Zwanziger action, in which the operator $\left(\bar{\varphi}_{\mu}^{a b} \varphi_{\mu}^{a b}-\bar{\omega}_{\mu}^{a b} \omega_{\mu}^{a b}\right)$ is taken into account from the beginning. This is achieved by adding to the Gribov-Zwanziger action the term

$$
S_{\bar{\varphi} \varphi}=-\int d^{4} x \mu^{2}\left(\bar{\varphi}_{\mu}^{a b} \varphi_{\mu}^{a b}-\bar{\omega}_{\mu}^{a b} \omega_{\mu}^{a b}\right)
$$

which is left invariant by the BRST transformations (3). The parameter $\mu^{2}$ is a mass parameter which, as much as the Gribov parameter $\gamma$, is fixed by a variational principle, see [1, 11] $]^{3}$. The refined Gribov-Zwanziger action is thus defined by

$$
S_{\mathrm{RGZ}}=S_{\mathrm{GZ}}+S_{\bar{\varphi} \varphi} .
$$

Since the term $S_{\bar{\varphi} \varphi}$ is BRST invariant, the action $S_{\mathrm{RGZ}}$ displays the same breaking term of the Gribov-Zwanziger action $S_{\mathrm{GZ}}$, i.e.

$$
\begin{aligned}
s S_{\mathrm{RGZ}}= & \int d^{4} x g f^{a b m}\left(D_{\nu}^{b p} c^{p}\right)\left(\left(\partial_{\nu} \bar{\varphi}_{\mu}^{a c}\right) \varphi_{\mu}^{m c}-\left(\partial_{\nu} \bar{\omega}_{\mu}^{a c}\right) \omega_{\mu}^{m c}\right) \\
& -\gamma^{2} g \int d^{4} x\left(c^{m} D_{\mu}^{m a}\left(f^{a b c}\left(\varphi_{\mu}^{b c}+\bar{\varphi}_{\mu}^{b c}\right)\right)+f^{a b c} A_{\mu}^{a} \omega_{\mu}^{b c}\right) .
\end{aligned}
$$

The refined Gribov-Zwanziger action turns out to be renormalizable to all orders, due to the large set of Ward identities which can be established [1, 11]. It is worth mentioning that the action (88) gives rise to a decoupling type solution for the ghost and gluon propagator. The ghost propagator turns out to behave as $1 / k^{2}$ in the infrared, while the gluon propagator does not vanish at the origin in momentum space, exhibiting positivity violation 4 , namely

$$
\left\langle A_{\mu}^{a}(p) A_{\nu}^{b}(-p)\right\rangle=\delta^{a b} \frac{p^{2}+\mu^{2}}{p^{4}+\mu^{2} p^{2}+2 g^{2} N \gamma^{4}}\left(\delta_{\mu \nu}-\frac{p_{\mu} p_{\nu}}{p^{2}}\right) .
$$

The infrared behavior displayed by expression (90) is in good agreement with the most recent numerical simulations performed on huge lattices [14, 15, 16, 17, 18], as well as with the analytical

\footnotetext{
${ }^{3}$ The parameter $\mu$ is determined by requiring that the Gribov no-pole condition [3] is fulfilled in the presence of the new operator (87), so that the Gribov horizon is not crossed. In other words, the introduction of the term (87) can be done in such a way that one remains within the Gribov region 1, 11]

${ }^{4}$ The positivity violation of the gluon propagator invalidates the interpretation of the gluons as excitations of the physical spectrum of the theory, providing a simple understanding of gluon confinement.
} 
results found in [19, 20]. Let us underline that, without the introduction of the operator (87), the Gribov-Zwanziger action would give rise to a different behavior for the propagators, namely to an enhanced ghost and to a gluon propagator which would vanish at the origin, see also the recent discussion presented in [21].

Let us proceed by showing how the operator $s_{\gamma}$ generalizes to the present case. From the refined Gribov-Zwanziger action, we get

$$
\begin{aligned}
\frac{\delta S_{\mathrm{RGZ}}}{\delta \bar{c}^{a}} & =-\mathcal{M}^{a b} c^{b}, \\
\frac{\delta S_{\mathrm{RGZ}}}{\delta \bar{\omega}_{\mu}^{a c}} & =\left(\mathcal{M}^{a b}+\mu^{2} \delta^{a b}\right) \omega_{\mu}^{b c},
\end{aligned}
$$

so that

$$
\begin{aligned}
c^{b}(x) & =-\left(\mathcal{M}^{-1}\right)_{x y}^{b c} \frac{\delta S_{\mathrm{RGZ}}}{\delta \bar{c}_{y}^{c}}=-\int d^{4} y\left[\left(\mathcal{M}^{-1}(x, y)\right)^{b c}\right] \frac{\delta S_{\mathrm{RGZ}}}{\delta \bar{c}^{c}(y)}, \\
\omega_{\mu}^{d c}(x) & =\left(\left(\mathcal{M}+\mu^{2}\right)^{-1}\right)_{x y}^{d a} \frac{\delta S_{\mathrm{RGZ}}}{\delta \bar{\omega}_{y \mu}^{a c}}=\int d^{4} y\left[\left(\left(\mathcal{M}+\mu^{2}\right)^{-1}\right)_{x y}^{d a}\right] \frac{\delta S_{\mathrm{RGZ}}}{\delta \bar{\omega}^{a c}(y)} .
\end{aligned}
$$

Thus, equation (89) can be rewritten as

$$
s S_{\mathrm{RGZ}}=\int d^{4} x\left(-\left(D_{\nu}^{m a} \Lambda_{\nu}^{a}\right)_{x}\left(\mathcal{M}^{-1}\right)_{x y}^{m c} \frac{\delta S_{\mathrm{RGZ}}}{\delta \bar{c}_{y}^{c}}-\gamma^{2} g f^{a b c} A_{\mu}^{a}(x)\left(\left(\mathcal{M}+\mu^{2}\right)^{-1}\right)_{x y}^{b m} \frac{\delta S_{\mathrm{RGZ}}}{\delta \bar{\omega}_{y \mu}^{m c}}\right),
$$

with $\Lambda_{\nu}^{a}$ given in expression (66). Eq.(93) expresses the exact invariance of the refined GribovZwanziger action $S_{\mathrm{RGZ}}$ under the nonlocal operator $s_{\gamma}^{(\mu)}$

$$
s_{\gamma}^{(\mu)} S_{\mathrm{RGZ}}=0
$$

where the operator $s_{\gamma}^{(\mu)}$ is now given by

$$
\begin{aligned}
s_{\gamma}^{(\mu)} A_{\nu}^{a} & =-\left(D_{\nu} c\right)^{a} \\
s_{\gamma}^{(\mu)} c^{a} & =\frac{1}{2} g f^{a b c} c^{b} c^{c} \\
s_{\gamma}^{(\mu)} \bar{c}^{a}(x) & =b^{a}(x)+\int d^{4} y\left(D_{\nu}^{m c} \Lambda_{\nu}^{c}\right)_{y}\left(\mathcal{M}^{-1}\right)_{y x}^{m a}, \\
s_{\gamma}^{(\mu)} b^{a} & =0 \\
s_{\gamma}^{(\mu)} \bar{\omega}_{\nu}^{a b}(x) & =\bar{\varphi}_{\nu}^{a b}(x)+\gamma^{2} g \int d^{4} y f^{m p b} A_{\nu}^{m}(y)\left(\left(\mathcal{M}+\mu^{2}\right)^{-1}\right)_{y x}^{p a}, \\
s_{\gamma}^{(\mu)} \bar{\varphi}_{\nu}^{a b} & =0 \\
s_{\gamma}^{(\mu)} \varphi_{\nu}^{a b} & =\omega_{\nu}^{a b} \\
s_{\gamma}^{(\mu)} \omega_{\nu}^{a b} & =0 .
\end{aligned}
$$

The same calculations performed in the previous sections with the operator $s_{\gamma}$ within the GribovZwanziger action, can be repeated by employing the operator $s_{\gamma}^{(\mu)}$ and by taking as starting action the refined Gribov-Zwanziger action. 


\section{Conclusion}

- In this work we have addressed the issue of the BRST symmetry in presence of the Gribov horizon. Our main results are expressed by equations (67) - (68), which show that, due to the positivity of the Faddeev-Popov operator $\mathcal{M}^{a b}$ within the Gribov region $\Omega$, the soft breaking of the BRST symmetry exhibited by the Gribov-Zwanziger action can be converted into an exact invariance. The resulting modified operator $s_{\gamma}$ displays an explicit dependence from the Gribov parameter $\gamma$. We have shown, through few examples, that the operator $s_{\gamma}$ might be helpful in order to evaluate the vacuum expectation value of quantities which are BRST exact.

As far as the renormalizability properties of the Gribov-Zwanziger action are concerned, the operator $s_{\gamma}$ has not to be considered as a substitute of the BRST operator s. Albeit broken, we remind here that the BRST transformations (3) give rise to a set of softly broken Slavnov-Taylor identities which are perfectly suitable for a study of the renormalizability of the theory within a local framework [6, 7, 9, 10, 11, 12. Rather, the operator $s_{\gamma}$ has to be seen as a useful device to extract non-perturbative Ward identities in order to characterize the vacuum expectation value of quantities which are BRST exact.

- It is worth spending here a few words about the Gribov-Zwanziger (GZ) action and the socalled refined Gribov-Zwanziger action (RGZ) introduced in [1, 11]. Both GZ and RGZ models are renormalizable and exhibit the same BRST soft breaking. Moreover, as it is apparent from the expression (87), the GZ and RGZ models differ by a BRST exact term, notably

$$
\left(\bar{\varphi}_{\mu}^{a b} \varphi_{\mu}^{a b}-\bar{\omega}_{\mu}^{a b} \omega_{\mu}^{a b}\right)=s\left(\bar{\omega}_{\mu}^{a b} \varphi_{\mu}^{a b}\right)
$$

As already mentioned, the introduction of this operator in the RGZ action is supported by the non-vanishing value of the condensate $\left\langle 0\left|\left(\bar{\varphi}_{\mu}^{a b} \varphi_{\mu}^{a b}-\bar{\omega}_{\mu}^{a b} \omega_{\mu}^{a b}\right)\right| 0\right\rangle$, whose non-perturbative effects are taken into account from the beginning, through the introduction of expression (96). We point out that the operator (96) is the only operator which can be added to the GZ action without jeopardizing the renormalizability, while keeping the same BRST breaking as well as the same field content. In the absence of the Gribov horizon, and thus in the case of an unbroken BRST symmetry, the two actions would be physically equivalent. However, in the present case in which the BRST symmetry is softly broken, they give rise to rather different predictions, both in four and three dimensions. In fact, while the GZ action predicts an infrared enhanced ghost sector and a gluon propagator which vanishes at the origin [21], the output of the RGZ action is a ghost propagator which is not enhanced in the infrared and a gluon propagator which does not vanish at the origin in momentum space. So far, the behavior predicted by the RGZ model seems to be in good agreement with the recent numerical data [14, 15, 16, 17, 18. Interestingly, in two dimensions, both models coalesce [22], giving an enhanced ghost and a vanishing gluon propagator, in agreement with the lattice data in two dimensions [23, 14, 18]. At present, we are unable to give a simple explanation of the different behavior predicted by the GZ and RGZ actions in three and four dimensions. We believe that a better understanding of the meaning of the BRST breaking and of its deep connection with the Gribov horizon is needed.

\footnotetext{
${ }^{5}$ This is due to fact that in $2 \mathrm{~d}$ the operator (96) cannot be safely introduced 22, due to the appearance of infrared divergencies in the Feynman integrals, typical of $2 \mathrm{~d}$ theories. We notice in fact that in $2 \mathrm{~d}$ the auxiliary fields $\left(\bar{\varphi}_{\mu}^{a b}, \varphi_{\mu}^{a b}, \bar{\omega}_{\mu}^{a b}, \omega_{\mu}^{a b}\right)$ are dimensionless.
} 
- Another issue which deserves further investigation is that of the role played by the auxiliary fields $\left(\bar{\varphi}_{\mu}^{a b}, \varphi_{\mu}^{a b}, \bar{\omega}_{\mu}^{a b}, \omega_{\mu}^{a b}\right)$ within the Gribov-Zwanziger framework. As already noticed, the introduction of these fields stems from the necessity of localizing the horizon function, eq.(1), so that a local and renormalizable action is obtained from the restriction to the Gribov region $\Omega$. These fields are thus expected to carry nontrivial information about the Gribov horizon, a fact which can be already inferred from the explicit dependence of the dimension two condensate $\left\langle\bar{\varphi}_{\mu}^{a b} \varphi_{\mu}^{a b}-\bar{\omega}_{\mu}^{a b} \omega_{\mu}^{a b}\right\rangle$ from the Gribov parameter $\gamma$, eq.(44). Also, as the restriction to the Gribov region deeply modifies the infrared behavior of the theory, we believe that a better understanding of the dynamics of the auxiliary fields could provide useful information about the confining character of the theory as well as about the construction of its physical spectrum. It is worth therefore to add here a few remarks on the on-going investigation [24] about the role which these fields might play. For illustration purposes, let us rely on an explicit example taken from the Gribov-Zwanziger theory, see [6]. Let us start by giving a look at the gluon propagator of the Gribov-Zwanziger theory, which can be obtained from the expression (90) by setting $\mu=0$, namely

$$
\left\langle A_{\mu}^{a}(k) A_{\nu}^{b}(-k)\right\rangle=\delta^{a b}\left(\delta_{\mu \nu}-\frac{k_{\mu} k_{\nu}}{k^{2}}\right) \frac{k^{2}}{k^{4}+\hat{\gamma}^{4}}, \quad \hat{\gamma}^{4}=2 g^{2} N \gamma^{4}
$$

As it is apparent, expression (97) displays complex poles at $k^{2}= \pm i \hat{\gamma}^{2}$, which invalidates the interpretation of gluons as excitations of the physical spectrum. In other words, in the infrared, gluons cannot be considered as part of the physical spectrum: they are confined. The physical excitations of the theory would correspond to colorless bound states of gluons, i.e. glueballs. We expect thus that information about the physical spectrum could be obtained by looking at the correlation function of colorless gauge invariant operators as, for example:

$$
G(p)=\int d^{4} x e^{-i p x}\left\langle F^{2}(x) F^{2}(0)\right\rangle
$$

where $F^{2}(x)=F_{\mu \nu}^{a}(x) F_{\mu \nu}^{a}(x)$. This correlation function is useful in order to investigate the properties of the scalar spin zero glueball state. Within the Gribov-Zwanziger theory, it has to be evaluated with the Feynman rules obtained by employing the confining gluon propagator (97). The explicit first order evaluation of expression (98) can be found in [6], and can be summarized as follows:

$$
G(p)=G^{\text {phys }}(p)+G^{\text {unphys }}(p) .
$$

The unphysical part, $G^{\text {unphys }}(p)$, displays cuts beginning at the unphysical values $p^{2}= \pm 4 i \hat{\gamma}^{2}$, whereas the physical part, $G^{\text {phys }}(p)$, has a cut beginning at the physical threshold $p^{2}=-2 \hat{\gamma}^{2}$. Moreover, the spectral function of $G^{\text {phys }}(p)$ turns out to be positive, so that it obeys the KallenLehmann representation [6]. As such, $G^{\text {phys }}(p)$ is an acceptable correlation function for physical glueball excitations. What is interesting in expression (99) is that a physical cut has emerged in the correlation function of gauge invariant quantities, even if it has been evaluated with a gluon propagator exhibiting only unphysical complex poles. This is precisely what one expects from a confining theory. Gluons are not physical excitations, but the glueball correlation function displays a physical singularity.

Of course, we have still to face the very difficult presence of the unphysical part $G^{\text {unphys }}(p)$. Here, we can argue that the presence of the auxiliary fields $\left(\bar{\varphi}_{\mu}^{a b}, \varphi_{\mu}^{a b}, \bar{\omega}_{\mu}^{a b}, \omega_{\mu}^{a b}\right)$ might be a welcome feature. In fact, we can figure out that, in the presence of the Gribov horizon, the correlation functions of the physical operators should receive contributions from the horizon, which could be 
accounted for by the auxiliary fields. This reasoning can be made on a quantitative basis by recalling that in ordinary Yang-Mills theories the construction of the physical operators is obtained through the cohomology of the nilpotent BRST operator [2, 25]. It is a remarkable fact that, in the present case, the BRST operator $s$ of eqs.(3) remains nilpotent, so that physical operators can be still associated to its cohomology classes. Furthermore, one should observe that the auxiliary fields $\left(\bar{\varphi}_{\mu}^{a b}, \varphi_{\mu}^{a b}, \bar{\omega}_{\mu}^{a b}, \omega_{\mu}^{a b}\right)$ are assembled in BRST doublets [25], so that they can only appear through terms which are BRST exact. Therefore, for the glueball operator $O_{g l u e b}(x)$ we would write

$$
O_{\text {glueb }}(x)=F^{2}(x)+s \mathcal{R}_{\gamma}(x),
$$

where $\mathcal{R}_{\gamma}$ is the exact BRST piece which depends on the auxiliary field®6. Expression (100) is easily seen to be BRST invariant, namely

$$
s O_{\text {glueb }}(x)=0 .
$$

For the glueball correlation function, we would get

$$
\left\langle O_{\text {glueb }}(x) O_{\text {glueb }}(y)\right\rangle=\left\langle F^{2}(x) F^{2}(y)\right\rangle+\langle s \Lambda(x, y)\rangle
$$

where $\Lambda(x, y)$ stands for

$$
\Lambda(x, y)=\mathcal{R}_{\gamma}(x) F^{2}(y)+F^{2}(x) \mathcal{R}_{\gamma}(y)+\mathcal{R}_{\gamma}(x) s\left(\mathcal{R}_{\gamma}(y)\right) .
$$

In the absence of the Gribov horizon, i.e. when $\gamma=0$, the inclusion of the BRST exact term $s \mathcal{R}_{\gamma}(x)$ is completely irrelevant, due to the fact the BRST symmetry is unbroken. As a consequence, $\langle s \Lambda(x, y)\rangle=0$, so that the correlator $\left\langle O_{\text {glueb }}(x) O_{\text {glueb }}(y)\right\rangle$ reduces to $\left\langle F^{2}(x) F^{2}(y)\right.$. However, in the presence of the Gribov horizon, i.e. for $\gamma \neq 0$, BRST symmetry is softly broken, so that the quantity $\langle s \Lambda(x, y)\rangle$ is now non-vanishing. We could thus search for a suitable term $\mathcal{R}_{\gamma}(x)$ which would enable us to cancel, order by order, the unphysical contributions contained in $G^{\text {unphys }}(p)$. Thus, the correlation function $\left\langle O_{\text {glueb }}(x) O_{\text {glueb }}(y)\right\rangle$ would display only physical poles, corresponding to colorless gluon bound states. At the present stage, such a possibility has to be regarded as an interesting plausible working hypothesis [24]. Certainly, it is a point worth to be investigated. If true, this would mean that the Gribov horizon combines in a nice and friendly fashion with the soft breaking of the BRST operator in such a way that the unphyiscal cuts present in the correlation functions of physical operators could be removed order by order. The presence of the auxiliary fields would be thus a quite welcome feature, as they would give us a way to construct the exact $\operatorname{BRST}$ term $\mathcal{R}_{\gamma}(x)$ needed to account for the unphysical cuts. At the same time, the existence of the operator $s_{\gamma}$ would enable us to evaluate the quantity $\langle s \Lambda(x, y)\rangle$ in a simple and efficient way.

- A topic which we also intend to analyse is that of the possible existence of a relationship between the non-local operator $s_{\gamma}$ and the color invariance of the Gribov-Zwanziger action. This could open a small window towards a better understanding of the color symmetry in the presence of the Gribov horizon.

- Finally, it is worth mentioning that the construction of the non-local operator $s_{\gamma}$ outlined here can be generalized to the maximal Abelian gauge [27, 28, 29, 30, 31, 32], for which a study of the properties of the corresponding Gribov region is available [33].

\footnotetext{
${ }^{6}$ See also the recent work [26].
} 


\section{Acknowledgments}

It is a pleasure to thank Laurent Baulieu, Attilio Cucchieri, David Dudal, Nele Vandersickel and Daniel Zwanziger for many interesting discussions. The Conselho Nacional de Desenvolvimento Científico e Tecnológico (CNPq-Brazil), the Faperj, Fundação de Amparo à Pesquisa do Estado do Rio de Janeiro, the SR2-UERJ and the Coordenação de Aperfeiçoamento de Pessoal de Nível Superior (CAPES), the CLAF, Centro Latino-Americano de Física, are gratefully acknowledged for financial support.

\section{References.}

[1] D. Dudal, S. P. Sorella, N. Vandersickel and H. Verschelde, New features of the gluon and ghost propagator in the infrared region from the Gribov-Zwanziger approach, Phys. Rev. D 77, 071501 (2008) arXiv:0711.4496 [hep-th]].

[2] C. Becchi, The renormalization content of Slavnov-Taylor identities, In 't Hooft, G. (ed.): 50 years of Yang-Mills theory, 168-185, World Scientific, 2005.

[3] V. N. Gribov, Quantization of non-Abelian gauge theories, Nucl. Phys. B 139, 1 (1978).

[4] K. Fujikawa, Dynamical Stability Of The Brs Supersymmetry And The Gribov Problem, Nucl. Phys. B 223, 218 (1983).

[5] D. Dudal, S. P. Sorella, N. Vandersickel and H. Verschelde, Gribov no-pole condition, Zwanziger horizon function, Kugo-Ojima confinement criterion, boundary conditions, BRST breaking and all that, arXiv:0904.0641 [hep-th].

[6] D. Zwanziger, Local And Renormalizable Action From The Gribov Horizon, Nucl. Phys. B 323, 513 (1989).

[7] D. Zwanziger, Renormalizability of the critical limit of lattice gauge theory by BRS invariance, Nucl. Phys. B 399, 477 (1993).

[8] P. van Baal, More (thoughts on) Gribov copies, Nucl. Phys. B 369, 259 (1992).

[9] N. Maggiore and M. Schaden, Landau Gauge Within The Gribov Horizon, Phys. Rev. D 50, 6616 (1994) arXiv:hep-th/9310111.

[10] D. Dudal, R. F. Sobreiro, S. P. Sorella and H. Verschelde, The Gribov parameter and the dimension two gluon condensate in Euclidean Yang-Mills theories in the Landau gauge, Phys. Rev. D 72, 014016 (2005) arXiv:hep-th/0502183.

[11] D. Dudal, J. A. Gracey, S. P. Sorella, N. Vandersickel and H. Verschelde, A refinement of the Gribov-Zwanziger approach in the Landau gauge: infrared propagators in harmony with the lattice results, Phys. Rev. D 78, 065047 (2008) [arXiv:0806.4348 [hep-th]].

[12] J. A. Gracey, One loop gluon form factor and freezing of alpha(s) in the Gribov-Zwanziger QCD Lagrangian, JHEP 0605, 052 (2006) arXiv:hep-ph/0605077. 
[13] L. Baulieu and S. P. Sorella, Soft breaking of BRST invariance for introducing nonperturbative infrared effects in a local and renormalizable way, Phys. Lett. B 671, 481 (2009) arXiv:0808.1356 [hep-th]].

[14] A. Cucchieri and T. Mendes, Constraints on the IR behavior of the gluon propagator in YangMills theories, Phys. Rev. Lett. 100, 241601 (2008) [arXiv:0712.3517 [hep-lat]].

[15] A. Cucchieri and T. Mendes, Constraints on the IR behavior of the ghost propagator in YangMills theories, Phys. Rev. D 78, 094503 (2008) [arXiv:0804.2371 [hep-lat]].

[16] V. G. Bornyakov, V. K. Mitrjushkin and M. Muller-Preussker, Infrared behavior and Gribov ambiguity in SU(2) lattice gauge theory, arXiv:0812.2761 [hep-lat].

[17] I. L. Bogolubsky, E. M. Ilgenfritz, M. Muller-Preussker and A. Sternbeck, Lattice gluodynamics computation of Landau gauge Green's functions in the deep infrared, arXiv:0901.0736 [hep-lat].

[18] A. Cucchieri and T. Mendes, Landau-gauge propagators in Yang-Mills theories at beta $=0$ : massive solution versus conformal scaling, arXiv:0904.4033 [hep-lat].

[19] A. C. Aguilar, D. Binosi and J. Papavassiliou, Gluon and ghost propagators in the Landau gauge: Deriving lattice results from Schwinger-Dyson equations, Phys. Rev. D 78, 025010 (2008) arXiv:0802.1870 [hep-ph]].

[20] Ph. Boucaud, J. P. Leroy, A. Le Yaouanc, J. Micheli, O. Pene and J. Rodriguez-Quintero, On the IR behaviour of the Landau-gauge ghost propagator, JHEP 0806, 099 (2008) arXiv:0803.2161 [hep-ph]].

[21] D. Zwanziger, Some exact infrared properties of gluon and ghost propagators and long-range force in QCD, arXiv:0904.2380 [hep-th].

[22] D. Dudal, S. P. Sorella, N. Vandersickel and H. Verschelde, The effects of Gribov copies in 2D gauge theories, arXiv:0808.3379 [hep-th].

[23] A. Maas, Two- and three-point Green's functions in two-dimensional Landau-gauge YangMills theory, Phys. Rev. D 75, 116004 (2007) arXiv:0704.0722 [hep-lat]].

[24] D. Dudal etal., work in progress.

[25] O. Piguet and S. P. Sorella, Algebraic renormalization: Perturbative renormalization, symmetries and anomalies, Lect. Notes Phys. M28, 1 (1995).

[26] D. Dudal, S. P. Sorella, N. Vandersickel and H. Verschelde, A renormalization group invariant scalar glueball operator in the (Refined) Gribov-Zwanziger framework, arXiv:0906.4257 [hepth].

[27] G. 't Hooft, Nucl. Phys. B 190 (1981) 455.

[28] A. S. Kronfeld, G. Schierholz and U. J. Wiese, Nucl. Phys. B 293 (1987) 461.

[29] H. Min, T. Lee and P. Y. Pac, Renormalization Of Yang-Mills Theory In The Abelian Gauge, Phys. Rev. D 32, 440 (1985). 
[30] A. R. Fazio, V. E. R. Lemes, M. S. Sarandy and S. P. Sorella, The diagonal ghost equation Ward identity for Yang-Mills theories in the maximal Abelian gauge, Phys. Rev. D 64, 085003 (2001) arXiv:hep-th/0105060].

[31] D. Dudal, J. A. Gracey, V. E. R. Lemes, M. S. Sarandy, R. F. Sobreiro, S. P. Sorella and $\mathrm{H}$. Verschelde, An analytic study of the off-diagonal mass generation for Yang-Mills theories in the maximal Abelian gauge, Phys. Rev. D 70, 114038 (2004) arXiv:hep-th/0406132.

[32] M. A. L. Capri, V. E. R. Lemes, R. F. Sobreiro, S. P. Sorella and R. Thibes, The gluon and ghost propagators in Euclidean Yang-Mills theory in the maximal Abelian gauge: taking into account the effects of the Gribov copies and of the dimension two condensates, Phys. Rev. D 77, 105023 (2008) [arXiv:0801.0566 [hep-th]].

[33] M. A. L. Capri, A. J. Gomez, V. E. R. Lemes, R. F. Sobreiro and S. P. Sorella, Study of the Gribov region in Euclidean Yang-Mills theories in the maximal Abelian gauge, Phys. Rev. D 79, 025019 (2009) arXiv:0811.2760 [hep-th]]. 